\title{
Ensayo de leche con bajo contenido de lactosa en diarrea aguda
}

\author{
M.C. Oscar Brunser T. ${ }^{1}$; M.C. Magdalena Araya Q. ${ }^{1} ;$ M.C. Julio Espinoza M. ${ }^{1}$ \\ M.C. Silvia Cruchet M. ${ }^{1}$; T.M. Isolda Pacheco M. ${ }^{1}$ \\ Whole powdered cow's milk and low lactose milk formula in \\ infants with acute diarrhea
}

\begin{abstract}
Fifty infants with acute diarrhea ( $\leqslant 5$ days of duration) were refed with either a low-lactose formula (experimental group, $N=25$ ) or whole powdered cow's milk (control group, $N=25$ ). During a two.month follow up etiology, clinical course, changes of anthroponsetsic parameters and tolerance to the milk products were evaluated. The etiology of diarrhoez, the mean duration of the episodes $(3.6 \pm 1.9$ and $3.9 \pm 1.9$ days in the experimental and control group, respectively) and the clinical course were comparable in both groups. Nutritional parameters remained unchanged during and after the episode. In two children $(8.3 \%)$ of the control group stools continued to be liquid, fecal $\mathrm{pH}$ was 5 and reducing substances were positive. They had to be refed with the low-lactose product to induce remission of the symptoms. Both products were well tolerated. These findings suggest that availability of low-lactose formulae may be advantagenous in the clinical management of infants with acute diarthea and evidence of lactose intolerance.

(Key words: infants, diarhea, low-lactose milk, lactose intolerance, nutritional status.)
\end{abstract}

Una de las consecuencias más importantes de la diarrea aguda es el deterioro de la capacidad de digerir y absorber nutrientes, como conse-

1. Unidad de Gastroenterologia, INTA, Universidad de Chile. cuencia del daño de la mucosa intestinal ${ }^{1,2}$. Las moléculas no absorbidas ejercen un efecto osmótico que aumenta el flujo de agua hacia el lumen, estimulando la peristalsis y acortando el tiempo de tránsito. La llegada de moléculas no absorbidas a los segmentos distales del intes- 
tino permite su fermentación por la flora residente. Parte de los productos resultantes de este proceso son absorbidos por la mucosa colónica, mientras que el resto puede inducir la aparición de diarrea osmótica ${ }^{3,4}$. Uno de los ejemplos mejor conocidos de esta situación es la mala absorción de lactosa. La relación entre enteropatógeno, lesión de la mucosa intestinal e intolerancia a hidratos de carbono es impredecible. La intolerancia clínicamente significativa agrava los efectos del episodio de diarrea sobre el estado nutricional ${ }^{\varsigma}$. La disponibilidad de una fórmula con bajo contenido de lactosa nos permitió evaluar el curso de los episodios de diarrea, así como la evolución del estado nutricional de nifios alimentados con ella.

\section{PACIENTES Y PROCEDIMIENTOS}

El estuđio se realizó en dos grupos de 25 lactantes cada uno, cuya edad era 6 a 12 meses al momento del ingreso. Los nifios eran regularmente controlados en el consultorio La Faena, perteneciente al Servicio de Salud Metropolitano Suroriente de Santiago, habian pesado $2.500 \mathrm{~g}$ o más al nacer, habían sido destetados espontáneamente, $y$ el episodio por el que consultaban ten ia una duración inferior a 5 días; además, no hab ian sido tratados con antibióticos y la deshidratación que presentaban se estimó que no exced ja el $7 \%$ del peso. Su peso/edad era superior al $80 \%$ del estándar NCHS ${ }^{6}$.

Durante la primera semana los niños fueron evaluađos 3 veces por el médico y las enfermeras del equipo de investigación, y hego, cada 15 días hasta completar 2 meses. En cada oportunidad se registró peso y talla y los resultados se expresaron como peso/edad, peso/talla y talla/edad, en relación al estándar NCHS. Durante la primera entrevista con la madre se completó un formulario, previamente codificado, que permitió evaluar el estrato socioeconómico 7,8 , abandono patemo, tipo y calidad de la vjvienda, disponibilidad de agua potable $y$ de facilidades sanitarias. Los padres de los niños que iban a participar en el estudio manifestaron por escrito su voluntad de incorporarse a éste. Las familias fueron visitadas dos veces por semana en sus casas por enfermeras de terreno. Durante las visitas se registró la alimentación recibida por el lactante: se obtuvieron muestras fecales para estudio etiológico del episodio de diarrea aguda y se evaluó la aceptabilidad de los productos ensayados.

Todos los niños se tehidrataron con una solución oral que proporcionaba $60 \mathrm{mEq}$ de $\mathrm{Na}, 20 \mathrm{mEq}$ de $\mathrm{K}$, $80 \mathrm{mEq}$ de $\mathrm{Cl}, 30 \mathrm{mEq}$ de bicarbonato y 111 mmoles de glucosa por 1 , los cuales se ofrecieron ad libitum durante las primeras 24 horas $^{9}$. La ingesta de una dieta pobre en fibra no se interrumpió, excepto en dos niños con vómitos iterativos. El producto con bajo contenido de lactosa ( $L L F$, Milupa $A G^{\circledR}$ ) se diluyó al $14 \%$ desde el inicio de la realimentación. Los niños del gnupo control recibieron leche de vaca en polvo, entera (Aptamil, Milupa AG()), en dilución al $5 \%$ el primer día. El volumen y, o, la concentración de estos ptoductos se aumentó según la tolerancia digestiva, de manera que el tercer día los aportes alcanzaban a cubrit los requerimientos para la edad. Su aceptabilidad fue evaluada al final de la primera semana $y$ al término del episodio en estudio, mediante un cuestionario especialmente diseñado para este efecto.

La etiología bacteriana se investigó mediante muestuas fecales obtenidas con dos tórulas el primer día y una al día siguiente. Los agentes buscados fueron: Escherichia coli enteropatogénica, sesotipos clásicos y cepas toxigénicas e invasoras. Shigella, Salmonella, Campylobacter y Yersinia ${ }^{10-14}$. La presencia de rotavirus se detectó por electroforesis del RNA aislado ${ }^{15}, 16$. El estudto parasitológico se efectuó en tres muestras fecales tomadas día por medio y procesadas de acuerdo a la técnica de Burrows ${ }^{17}{ }^{18}$. En base al criterio clínico, en aquellos casos en que se estimó necesario se practicaron pruebas de laboratorio adicionales.

Los resultados sc registraron en formularios precodificados y su análisis computacional se efectuó utilizando el paquetc esiadístico SAS.

\section{RESULTADOS}

Se incorporaron 53 niños al estudio, de los cuales 48 (24 en cada grupo) lo completaron. Los motivos de deserción fueron: problemas psiquiátricos de la madre (uno), cambio de domicilio (uno), mala aceptabilidad del producto control (uno). En dos niños del grupo control la aparición de intolerancia a la lactosa determinó su exclusión del protocolo y la necesidad de realimentarlos con el producto sin lactosa.

Los dos grupos de estudio resultaron comparathles para estrato socioeconómico, calidad de la vivienda, número total de miembros de la familia; edad, escolaridad y trabajo de ambos padres; hacinamiento y disponibilidad de facilidades sanitarias, peso y talla de nacimiento y edad y estado nutricional al inicio de la investigación.

Antes de ingresar al estudio las madres habian aumentado el volumen de líquidos of recidos en base a infusiones de hierbas y agua de arroz, ambas con azúcar. Ninguna habia usado una fórmula que contuviera sales. Muy pocas de ellas habían suspendido la alimentación de lácteos o sólidos. En la tabla 1 se muestra la mediana y el rango de los volúmenes de ingesta diaria en ambos grupos de estudio. La tabla 2 muestra la evaluación de la adecuación de la ingesta de alimentos sólidos durante los primeros 7 días. Se observa un rápido inçremento, tanto en el volumen de fórmula ingerido como de la proporción de niños, cuya ingesta de sólidos fue adecuada. 
Tabla 1

Ingesta de las fórmulas experimental y control en lactantes con diarrea aguda $(\mathrm{ml} / \mathrm{kg} / \mathrm{d}$ ia)

\begin{tabular}{lcccr}
\hline & $\begin{array}{c}\text { Grupo experimental } \\
\text { (leche con bajo } \\
\text { contenido de lactosa) }\end{array}$ & \multicolumn{2}{c}{$\begin{array}{c}\text { Grupo control } \\
\text { (leche con lactosa) }\end{array}$} \\
\hline Día & Mediana & Rango & Mediana & Rango \\
\hline 1 & 32 & $6-64$ & 37 & $8-112$ \\
2 & 52 & $14-137$ & 62 & $12-141$ \\
3 & 75 & $37-170$ & 84 & $15-177$ \\
5 & 80 & $59-170$ & 79 & $15-148$ \\
7 & 80 & $64-167$ & 90 & $15-141$ \\
\hline
\end{tabular}

Al ingreso ambos grupos también fueron comparables para las siguientes caracteristicas clínicas: presencia de vómito, fiebre, dolor y distensión abdominal, aspecto y número de las deposiciones, presencia de síntomas de otros sistemas y aparatos, etiología (tabla 3) y afecciones intercumentes durante el periodo de observación. La aceptabilidad de ambos productos fue
Tabla 2

Evaluación de la ingesta de sólidos en lactantes con diarrea aguda.

(Porcentajes de los nitios en control)

\begin{tabular}{|c|c|c|c|}
\hline Día & $\begin{array}{l}\text { Calidad de la } \\
\text { ingesta }\end{array}$ & $\begin{array}{l}\text { Grupo experimental } \\
\text { (leche con bajo } \\
\text { contenido de lactosa) }\end{array}$ & $\begin{array}{l}\text { Grupo control } \\
\text { (leche } \infty \text { n } \\
\text { lactosa) }\end{array}$ \\
\hline \multirow[t]{2}{*}{1} & adecuaća & $25^{2}$ & $25^{2}$ \\
\hline & inadecuada & $70^{2}$ & $55^{2}$ \\
\hline \multirow[t]{2}{*}{2} & adecuada & 35 & 55 \\
\hline & inadecuada & s5 & 20 \\
\hline \multirow[t]{2}{*}{3} & adecuada & $5 \mathrm{~S}$ & 45 \\
\hline & inadecuada & 40 & 30 \\
\hline \multirow[t]{2}{*}{5} & adecuada & 65 & 65 \\
\hline & inadecuada & 25 & 10 \\
\hline \multirow[t]{2}{*}{7} & adecuada & 85 & 65 \\
\hline & inadecuada & 5 & 10 \\
\hline
\end{tabular}

1 = Ingesta adecuada significa que el paciente ingirió cantidades adecuadas o que exced ían las reoomendaciones para la edad.

$2=$ Las vifras no suman $100 \%$ porque algunos niños aún no recibían sólidos.

Tabla 3

Enteropatógenos detectados en lactantes con diarrea aguda, alimentados con una fórmula con bajo contenido de lactosa (Grupo experimental) y leche de vaca (Grupo control)

Estudio bacteriológico

Grupo experimental
$\begin{gathered}\text { Grupo control } \\ \text { de lactos }\end{gathered}$$\quad$ Total
de lactoso)
Positivo

E. coli serotipos clásicos

Shigella

Campulobacter jejuni/coli

Yersinia encerocolftica

Negativo

TOTAL

8
12
20

\begin{tabular}{ll}
6 & \\
1 & \\
1 & \\
1 & \\
& 11 \\
\hline & 20
\end{tabular}

3

1
9

4

2

2

$\frac{23}{40}$

Rotavitus

Positivo

$\frac{2}{18}$

$\begin{array}{r}2 \\ 18 \\ \hline 20\end{array}$

$-\frac{4}{46}$

Enteroparásitos

Positivo

Giardia lamblia

Entamoeba histolytica

Cryptosporidium sp.

Negativo

0

2

l

TOTAL
3

3
1
0

4

3

3

1 
muy buena, tanto al comienzo como durante el estudio. El efecto de la intervención se evaluó a través de la duración del episodio de diarrea y de los cambios de los parámetros nutricionales. Respecto del primero, la duración media de los episodios fue $3,6 \pm 1,9$ y $3,9 \pm 1,9$ días $(\mathrm{p}=0,57)$ en el grupo experimental y control, respectivamente. En dos casos $(8,3 \%)$ que recibian la leche control (con lactosa) la diarrea continuó, y se demostró $\mathrm{pH}$ fecal bajo y presencia de sustancias reductoras en las heces. Los sintomas desaparecieron y los exámenes se normalizaron cuando ambos pacientes recibieron la leche sin lactosa (LLF). No se detectaron casos de diarrea persistente. El análisis del estado nutricional, expresado como porcentaje del percentil 50 del estándar NCHS para peso/edad, no mostró diferencias significativas entre ambos grupos de estudio durante los 60 días de observación (tabla 4). Comparando los resultados expresados como purcentaje de incremento o pérdida de la relación peso/taila en los distintos momentos de medición, tampoco se encontraron diferencias entre los grupos, ni dencro de cada uno de ellos.

\section{DISCUSION}

En este tipo de investigación es importante demostrar que los grupos en estudio son comparables con respecto a las variables intervinien-

\section{Tabla 4}

Relación Peso/Edad' promedio de niños con diarrca aguda que recibian leche con o sin lactosa)

\begin{tabular}{rcc}
\hline Día & $\begin{array}{c}\text { Grupo experimental } \\
\text { (leche con bajo contenido } \\
\text { de lactosa) }\end{array}$ & $\begin{array}{c}\text { Grupo control } \\
\text { (ieche con } \\
\text { lactosa) }\end{array}$ \\
\hline 1 & $96,70^{2}$ & 95,30 \\
3 & 95,95 & 94,72 \\
5 & 95,80 & 95,00 \\
15 & 96,15 & 96,10 \\
30 & 97,10 & 96,05 \\
45 & 96,95 & 95,80 \\
60 & 97,42 & 95,74 \\
\hline
\end{tabular}

3 = Los valores expresados como porcentaje del percentil $\mathbf{5 0}$ del estándar NCHS.

2 = No hubo diferencias significativas entre los grupos en ningún momento de la evolución, ni dentro de cada grupo. tes ${ }^{19}$. El análisis de la información recolectada en estos dos grupos de lactantes reveló que, efectivamente, esto fue así. Es interesante señalar que, previo al ingreso al protocolo, las madres habian administrado a los niños, es. pontáneamente, infusiones de hierbas o bien agua de arroz. Estos resultados son semejantes a los obtenidos en varios estudios anteriores $^{20,21}$, lo que nos lleva a sugerir la necesidad de educar a nuestra población para que utilicen soluciones que además contengan electrólitos ${ }^{y}$. Durante los últimos años se ha llegado a un consenso acerca de la conveniencia de mantener la alimentación o realimentar rápidamente a los pacientes con diarrea ${ }^{9}$. En este estudio se estableció como meta cubrir los requerimientos nutricionales de los pacientes al tercer dia de ingreso al protocolo, lo que puede parecer difícil de cumplir. El análisis individual de la ingesta de leche y sólidos (cuyos promedios para los grupos aparecen en las tablas 1 y 2 ) muestra que la in gesta de energía y nutrientes fue adecuada, incluso en aquellos pacientes en que la ingestión de lactosa inducía diarrea. Esto puede ser resultado del hecho de que se motivó a las madres para que suministraran alimentos en cantidad $y$ calidad adecuadas.

La disponibilidad de productos con bajo contenido de lactosa es ventajosa en el manejo de enfermos con diarrea aguda, ya que un porcentaje de éstos vuelve incapaz de digerir este disacárido ${ }^{22}$. La proporción de pacientes que sufren el trastorno es variable y algunas etiología parecen tener más posibilidades de producirlo que otras. Los niffos que presentan intolerancia a la lactosa y que continúan recibiendo este hidrato de carbono sufren diarreas prolongadas y eventualmente se desnutren'. En el caso de los lactantes, otro requerimiento de la fórmula es que debe sumjnistrar nutrientes en cantidad y calidad tales que permitan que el crecimiento continúe en forma satisfactoria. En este sentido, la fórmula sin lactosa usada en este estudio llena estos requisitos.

En investigaciones realizadas en la misma áres. $12 \%$ de los episodios de diarrea prolongada se asociaron a intolerancia a lactosa ${ }^{23}$. En otras regiones del mundo se ha demostrado que la intolerancia es más frecuente en lactantes desnutridos ${ }^{5}$. Sin embargo, conviene tener en cuenta que muchos de estos estudios se realizaron en grupos racjales, en los que la intolerancia de aparición tardía es muy frecuente ${ }^{24}$. 
A1 igual que en trabajos previos, también efectuados mediante el seguimiento de cohortes en terreno, en esta investigación la presentación clínica y la evolución de los síntomas de los pacientes no guardó relación con la etiologia detectada en asociación al episodio ${ }^{15,20,21}$.

Ambos productos ensayados tuvieron buena aceptabilidad, to que se tradujo en una proporción más baja que la habitual ( 1 caso) de abandonos voluntarios del estudio debido a rechazo de las fórmulas utilizadas. Esto, unido a la realjmentación precoz y enérgica podría explicar el escaso impacto nutricional que tuvieron los episodios de diarrea aguda (tabla 4 ).

\section{RESUMEN}

Se estudiaron 50 lactantes con diatrea de menos de 5 días de evolución, de los cuales la mitad se realimentó con una leche con bajo contenido de lactosa (grupo experimental) y los restantes con una fórmula láctea en polvo (grupo control). Durante un seguimiento de 2 meses se evaluaron el curso clínico, los cambios antropométricos y la tolerancia a los productos. La evolución clínica, las etiologías y la duración promedio de los episodios $(3,6 \pm 1,9$ y $3,9 \pm 1,9$ días en experimentales $y$ controles, respectivamente) fueron comparables en ambos grupos. No se observó deterioro nutricional durante ni después del episodio. En 2 nifios $(8,3 \%)$ del grupo control fue necesario administrar la leche sin lactosa para que remitieran los síntomas. La aceptabilidad a ambos productos fue satisfactoria. Estos resultados sugieren que la utilización de productos con bajo contenido de lactosa puede ser ventajosa en el manejo clínico de lactantes con diarrea aguda $y$ evidencia de intolerancia por lactosa.

(Palabras claves: diarrea aguda, leche pobre en lactosa, intolerancia a lactosa.)

\section{AGRADECIMIENTOS}

Deseamos agradecer la ayuda prestada por la Dra. Utsula Wachtel y Milupa A.G., Frjedrichsdorf, R.F.A. por proporcionar los productos ensayados $y$ por el apoyo a este proyecto de investigación; a la Dra. T. Lobos, Jefe del Laboratorio Clínico de la Clínica Las Condes, al Dr. J. García Moreno del Instituto de Salud Pública, Sra. A.M. Altieri y Dr. E. Spencer por los estudios etiológicos; a la Dra. L. Toro, Directora del Consultorio La Faena, y a la Srta. J. Abbate por su ayuda como se. cretaria.

\section{REFERENCIAS}

1. Chen L.C.: Interactions of diarrhea and manutrition. In: L.C. Chen and N.S. Scrimshaw, eds Diarthea and malnutrition. Interactions, mechanisms and interventions. New York: Plenum Press. 1983; 3-19.

2. Watsh J.A., Warren K.S. Selective primary health tare: an interim strategy for disease control in developing countries. $N$ Engl I Med 1979; 301: $967-976$.

3. Molla A.M., Molla A., Sarker S.A., Rahamon M.M.: Food intake during and after recovery from diarthea in children. In: L.C. Chen and N.S. Scrimshaw, eds. Diarrhea and Malnutrition. Interactions, mechanisms and interventions. New York: Plenum Ptess 1983; $113-123$.

4. Bond J.R., Currier B., Buchwald H. Lewit M.D.: Colonic conservation of malabsorbed carbohydrates. Gastroenterology 1980; 78: 444447.

5. Lifshitz $F$.: Perspective of carbohydrate intolerance in infants with diarrhea. En: Lifshitz, ed. Carbohydrate intolerance in infancy. New York: Marcel Dekker, Inc. 1982; 3-19.

6. Homill P.V., Drizd T.A., Johnson Ch., Reed R.D., Roche A.F., Moore W.M.: Physical growth: National Center for Health Statistics Percentiles. Am J Clin Nutr 1979; 32: 607-629.

7. Graffar $M$.: Etudes d'agglomération en cinq cent fanilles d'une commune de l'agglomeration bruxelloise. Laboratorie de Médicine Sociale. Brussels: Université Libre de Bruxelles, 1957.

8. Alvarez M.L., Wurgaft F, Salazar M.E.: Mediciones de nivel socioeconómico urbano en familias con lactantes desnutridos. Arch Latinoamer Nuts 1982; 32 : 325-331.

9. Programme for Control of Diarrhoogl Diseases. A manual for the treatment of acute diarhoea. For use by physicians and other senior health workers. WHO/CDD/SER/80. 2 Rev 1, 1984.

10. Mortin W.J., Washington IIJA.: Enterobacteriaceae. In: Lenette E.H., ed. Manual of clinical microbiology, $3^{\text {rd }}$ ed. Washington D.C.: American Society for Microbiology 1980; 195-219.

11. Butzler J.P., Skirrow M.B.: Campylobacter esteritis. Clin Gastroenterol 1979; 8: 737-765.

12. Ristaino P., Levine $M . M$., Young $C$.: Improved GM $\mathbf{M}_{1}$ enzyme-dinked immunosorbent assay for detection of Escherichia coli heat-labile enterotoxin. J Clin Microbiol 1983; 18: 808-815.

13. Gianella $R$.A.: Suckling mouse model for detection of heat-labile Escherichia coli enterotoxin: characteristics of the model. Infect Inumun 1976; 14: 95-99.

14. Sereny 8.: Experimental Shigella kerato-conjuntivitis. A preliminary report. Acta Microbiol Acad Sci Hung 1955; 2: 293-296.

15. Laemmli U.K.: Cleavage of structural protejns during the assembly of the head of bacteriophage T4. Nature $1970 ; 227: 680-685$.

16. Spencer E., Avendoño F, Garcia B.: Analysis of human rotavirus mixed electropherotypes. Infect Immun 1983: 39: 569-574. 
17. Burrows R.B.A.: A new fixative technique for the diagnosis of intestinal parasites. Am J Clin Pathol $1967 ; 48: 342-346$.

18. Sagua $H$ : Comparación del rendimiento de 3 métodos en el diagnóstico coproparasitológico. Rev Med Chile 1975; 103: 175-177.

19. Kleinbaum D.G., Kupper L.L., Morgenstem H.: lipidemiologic research. Principles and Quantitative Methods. New York: Van Nostrand Reinhold Company, 1982.

20. Brunser O. Araya M. Espinoza J. Guesry P.R., Secretin M.C., Pacheco I.: Effect of an acidified milk on diarhoeal disease and the carrier state in infants of the low socio-economic stratum. Acta Paediatr Scand 1989; $78: 259-264$.

21. Cordero P., Araya M., Espinoza J., Figueroa G., Pacheco 1., Brunser $O$.: Efecto de la hidratación oral y realimentación precoz en la evolución de la diarrea aguda del lactante. Rev Chil Podiati $1985 ; 56: 411-417$.

22. Anderson Ch. M., Grocey M.: Disorders of carbohydrate digestion and absorption. In: Ch. $M$. Anderson, V. Burke and M. Gracey, eds. Paediatric gastrocnterology, $2^{\text {nd }}$ edition. London: Blackwell Scientific Publications 1987; 353-374.

23. Araye $M$ : Diarrea prolongada. Rev Chil Pediatr 1988:59 (Suppl 2): 12-14.

24. Ransome-Kuti O.: Lactose intolerance. En: M. Gracey, ed. Diarrhoeal disease and malnutrition. Churchill livingstone: Londres. $1985 ; 102-118$.

25. Araya M., Figuerod $G$., Espinoza J, Montesinos N., Spencer E., Brunser $O$. . Acute diarrhoeal $^{\circ}$ disease in children under 7 years of age in a perjurban slum of Santiago, Chile. J Hyg Camb 1985; 95: $457-467$ 